\title{
Review \\ Endosomal Recycling Defects and Neurodevelopmental Disorders
}

Shinji Saitoh

check for

updates

Citation: Saitoh, S. Endosomal

Recycling Defects and

Neurodevelopmental Disorders. Cells 2022, 11, 148. https://doi.org/

$10.3390 /$ cells 11010148

Academic Editors: Koh-ichi Nagata and Orly Reiner

Received: 16 November 2021

Accepted: 1 January 2022

Published: 3 January 2022

Publisher's Note: MDPI stays neutral with regard to jurisdictional claims in published maps and institutional affiliations.

Copyright: (C) 2022 by the author Licensee MDPI, Basel, Switzerland. This article is an open access article distributed under the terms and conditions of the Creative Commons Attribution (CC BY) license (https:// creativecommons.org/licenses/by/ $4.0 /)$.
Department of Pediatrics and Neonatology, Graduate School of Medical Sciences, Nagoya City University, Kawasumi 1, Mizuho-cho, Mizuho-ku, Nagoya 467-8601, Japan; ss11@med.nagoya-cu.ac.jp; Tel.: +81-52-853-8246

\begin{abstract}
The quality and quantity of membrane proteins are precisely and dynamically maintained through an endosomal recycling process. This endosomal recycling is executed by two protein complexes: retromer and recently identified retriever. Defects in the function of retromer or retriever cause dysregulation of many membrane proteins and result in several human disorders, including neurodegenerative disorders such as Alzheimer's disease and Parkinson's disease. Recently, neurodevelopmental disorders caused by pathogenic variants in genes associated with retriever were identified. This review focuses on the two recycling complexes and discuss their biological and developmental roles and the consequences of defects in endosomal recycling, especially in the nervous system. We also discuss future perspectives of a possible relationship of the dysfunction of retromer and retriever with neurodevelopmental disorders.
\end{abstract}

Keywords: endosomal recycling; retromer; retriever; Schaaf-Yang syndrome; Ritscher-Schinzel syndrome

\section{Overview of the Endosomal Recycling System}

Membrane proteins play a fundamental role in various cellular functions, including cellular adhesion, nutrient uptake, and signal transduction [1]. The quality and quantity of these proteins are precisely and dynamically maintained through a recycling process [2]. Membrane proteins are constantly internalized through endocytosis and transferred to an early endosome. The fate of the early endosome varies - through endosomal trafficking, it may be recycled to the cell membrane or degraded in lysosomes (Figure 1). The endosomal recycling system is thus crucial for maintaining functional membrane proteins. Defects in endosomal recycling have significant effects on cellular function. For example, the regular exchange of membrane proteins is essential for maintaining synaptic plasticity in neurons [3]. Therefore, defects in endosomal recycling could cause human disorders, including neurological diseases.

Two protein complexes, retromer and recently identified retriever, are involved in recycling pathways $[4,5]$. In this review, we discuss the role of these two recycling complexes and the consequences of defects in endosomal recycling. Recent identification of neurodevelopmental disorders caused by pathogenic variants in genes associated with retriever has expanded the significance of endosomal recycling in neurodevelopmental disorders as well as neurodegenerative disorders. Thus, we discuss future perspectives of a possible relationship of the dysfunction of retromer and retriever to neurodevelopmental disorders. 


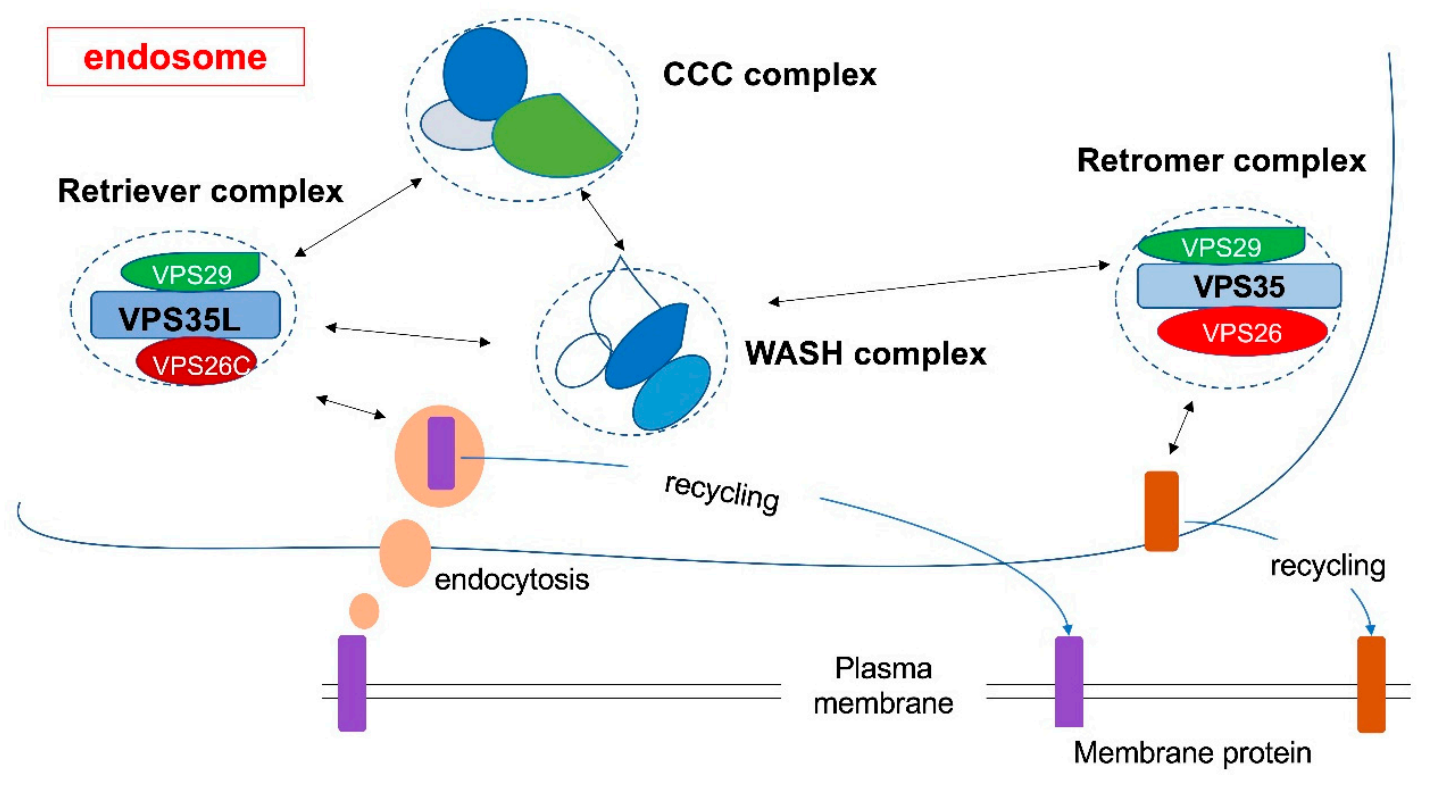

Figure 1. Schematic view of the endosome recycling system. Retromer and retriever complexes play a fundamental role in the recycling of numerous membrane proteins, although their target proteins are distinct. Retromer and retriever complexes interact with the WASH complex and the CCC complex for endosomal recycling (see text). WASH complex has an endosomal actin-remodeling function and is required for retromer and retriever to localize to the recycling endosome, while the CCC complex is shown to interact only with retriever.

\section{Retromer Complex}

Retromer was originally identified in yeast as a complex required for retrograde trafficking of vacuolar cargo [6]. Retromer recycles cargo to both the trans-Golgi network (TGN) and the plasma membrane in all eukaryotes. It is a heterotrimer complex composed of three subunits: VPS35, VPS29, and VPS26 [7]. Two paralogues, VPS26A and VPS26B, are expressed in humans and play distinct roles. VPS35 binds to VPS26 at the N-terminal and to VPS29 at the C-terminal. Retromer is enriched on the cytosolic face of the early and late endosome. It uses various sorting nexin (SNX) proteins as cargo adaptors. Binding to SNX3 mediates the localization of retromer in the early endosome, while RAS-related protein RAB7-GTP localizes retromer in the late endosome. Sequence-specific cargo recognition of retromer is partly mediated by SNX27 [8]. SNX27 recognizes $\Phi x N P x p Y$ or $\Phi x N x x p Y$ as a sorting motif (where $\Phi$ is a hydrophobic residue and $\mathrm{x}$ is any residue) at the carboxyterminal FERM-like domain. SNX27 also binds to a PDZ domain-binding sorting motif at the amino-terminal PDZ domain. More than 400 cargo proteins require SNX27 and retromer for their recycling $[9,10]$. Such cargo includes receptors and transporters that are essential for signal transduction, synaptic plasticity, and nutrient uptake.

\section{Retriever Complex}

Recently, a second retromer-like protein complex involved in endosomal recycling was identified and named retriever [5]. The retriever also forms a heterotrimer complex, and subunits are composed by VPS35L, VPS29, and VPS26C [4]. The structures of retromer and retriever are believed to be similar, although the precise structure of the retriever has not been determined. The retriever associates with SNX17, which recognizes $\Phi \times N P x Y$ or $\Phi \times N x x Y$ as a sorting motif [11]. SNX17 lacks the PDZ domain and thus does not associate with the proteins containing the PDZ-binding motif. The retriever binds to the COMMD/CCDC22/CCDC93 (CCC) complex and forms the commander complex, which is implicated in the trafficking of a variety of membrane proteins [12]. The commander complex interacts with the Wiskott-Aldrich Syndrome Protein and SCAR Homolog (WASH) complex, which has an endosomal actin-remodeling function, and with SNX17. The WASH 
complex also interacts with retromer for endosomal recycling, although the commander complex is thought to interact only with retriever [4]. Target proteins of the commander complex include the ATP7A/ ATP7B copper transporter, low-density lipoprotein receptor (LDLR) family proteins, and $\alpha 5 \beta 1$ integrin [12].

\section{Relationship between Retromer and Retriever}

Thus far, retromer and retriever are the only complexes proven to be involved in membrane protein recycling. Because of the architectural similarity, both complexes are thought to have evolved to play overlapping roles in membrane protein recycling. Indeed, VPS29 is shared in both complexes. The expression patterns of retromer and retriever in tissues, including brain, mostly overlap, although information is limited. Therefore, distinct roles of retromer and retriever appear to be determined by their target proteins. The specificity of target proteins is determined by adaptor proteins SNX27 and SNX17, which bind to VPS35 and VPS35L, respectively. As described above, SNX27 recognizes ФxNPxpY or $\Phi x N x x p Y$ as a sorting motif, while SNX17 recognizes $\Phi x N P x Y$ or $\Phi x N x x Y$. Therefore, retromer and retriever complexes recycle different membrane proteins based on the sequences of the sorting motif. For example, $\alpha \beta$ integrin is only recycled by retriever but not by retromer [5]. Nevertheless, since many membrane proteins interact, a dysfunction of retromer or retriever could affect overlapping proteins directly or indirectly [5]. Revealing the functional interactions of retromer and retriever complexes in future studies is of great interest.

\section{Human Disorders Associated with Retromer Dysfunction}

Retromer dysfunction is involved in several human diseases, in particular, neurodegenerative disorders including Alzheimer's disease (AD) and Parkinson's disease (PD) (Figure 2). Since endosomal recycling is important in synaptic plasticity and maintenance of neuronal health, neurons are highly vulnerable to a dysfunction of endosomal recycling.

Retromer or Retriever related ND?
Schaaf-Yang syndrome: MAGEL2
DD/ID
Autism
Hypotonia
Joint contracture
Prader-Willi syndrome: 15q11-q13
imprinted genes
DD/ID
Hypotonia
Hyperphagia
Hypogonadism
Hao-Fountain syndrome: USP7
DD/ID
Autism
Hypotonia

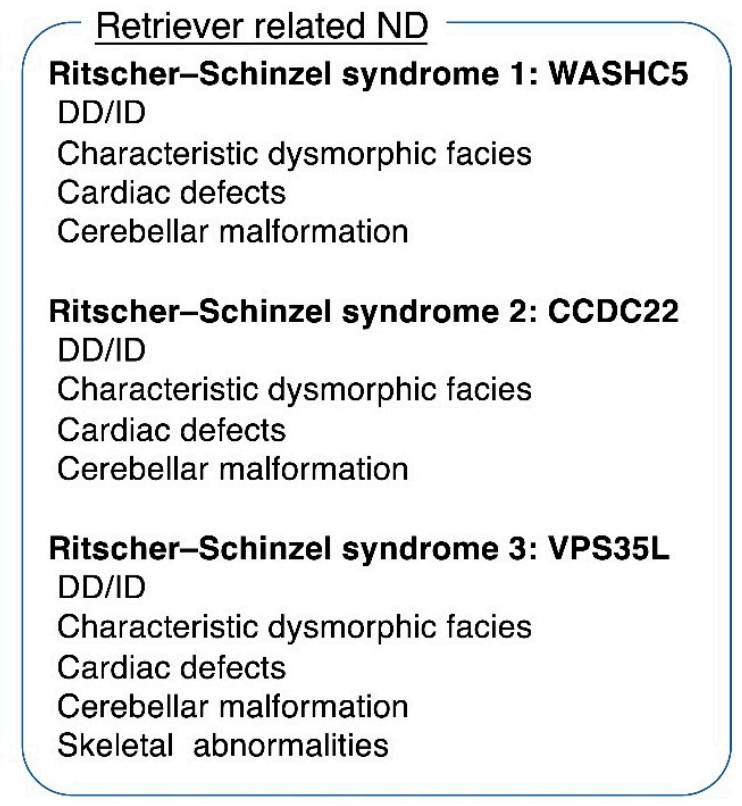

Figure 2. Putative neurodevelopmental disorders associated with retromer and retriever complexes. Major clinical features are demonstrated for each disorder. ND, neurodevelopmental disorder; DD, developmental delay; ID, intellectual disability.

The link between AD and retromer was first found in 2005. Using postmortem brains from AD patients and controls, Small et al. discovered that two retromer subunits, VPS26 and VPS35, were deficient in brains from patients with AD [13]. Subsequently, a line of genetic studies revealed the link between AD and retromer-related genes. Examples include 
SORL1, SNX1, SNX3, and RAB7A [14]. As described above, SNX3 and RAB7A encode major proteins binding to retromer.

The link between PD and retromer was first demonstrated by whole-exome sequencing. In 2011, autosomal dominant mutations in VPS35 were identified in patients with late-onset PD $[15,16]$. Subsequent studies revealed that the mutations caused a reduction in VPS35 binding to the WASH complex, and thereby induced $\alpha$-synuclein aggregates, which have a pathogenic role in PD, although non-neuronal cells were used for the experiments $[17,18]$. It is of interest that most risk or causal genes of PD are involved in autophagy-lysosomal-endosomal pathways. Dysfunction of these pathways therefore might induce an accumulation of $\alpha$-synuclein, which could be associated with the development of PD. However, the precise pathophysiology of VPS35 mutations in PD remains to be elucidated, and further investigation is necessary.

A dysfunction of retromer is also suggested in Down syndrome (DS) [19]. Individuals with DS have an increased risk of developing AD: more than $60 \%$ of individuals with DS will show clinical features of AD by 40 to 50 years of age [20]. Importantly, the neuropathological findings in $\mathrm{DS}$ are in line with those of $\mathrm{AD}$, as accumulating amyloid- $\beta(\mathrm{A} \beta)$ is found in elder individuals with DS who show cognitive decline as well as in patients with AD. This might be related to the fact that the A $\beta$ precursor protein (APP) gene is located on chromosome 21. A $\beta$ is first accumulated in the endosome system in individuals with DS as well as in those with $\mathrm{AD}$, indicating that endosomal trafficking including retromer is likely to play an important role in the clearance of $A \beta$ [21]. Recently, dysregulation of the retromer complex was reported in postmortem brain tissues and fibroblasts derived from individuals with DS [22]. Although the cause of dysregulation of the retromer complex in DS remains to be uncovered, dysfunctional endosomal recycling may play a significant role in the development of AD in individuals with DS.

Although the link between retromer dysfunction and AD, PD, or DS has been intensively studied, a pathogenic role for retromer in neurodevelopmental disorders remains to be uncovered. Although it is yet to be investigated, a putative example could be SchaafYang syndrome (SYS). SYS is a congenital disorder characterized by developmental delay, autism spectrum disorder, and multiple congenital joint contractures [23,24]. SYS is known to resemble Prader-Willi syndrome (PWS), which is a distinct developmental disease, particularly beyond infancy [25]. SYS is caused by a mutation in MAGEL2, which is a maternally imprinted gene located in the 15q11-q13 chromosome region [23]. MAGEL2 encodes a protein expressed predominantly in the brain. MAGEL2 protein binds the E3 RING ubiquitin ligase TRIM27 as well as USP7, which is a deubiquitinating enzyme and is recruited to the endosome through interaction with the WASH complex [26]. This complex is named the MUST (MAGEL2-USP7-TRIM27) complex. The MUST complex may facilitate the retromer endosomal recycling pathway through ubiquitination and activation of the WASH complex, which interacts with retromer as well as with retriever [4]. Therefore, MAGEL2 mutations reduce the function of the MUST complex and subsequently perturb endosomal recycling, which may underlie the pathophysiology of SYS. Some patients with SYS show neurological regression resembling encephalopathy after febrile infectious diseases [25]. Since retromer dysfunction is related to neurodegenerative disorders, it is of interest whether this acute neurological dysfunction in patients with SYS in febrile conditions is related to endosomal recycling dysfunction. Under stress conditions, including febrile episodes, neurons may need to adopt a rapid turnover of membrane proteins. Therefore, partial dysfunction of endosomal recycling might manifest as neurological dysfunction under critical conditions only. Therefore, not only neurodegeneration but intermittent neurological dysfunction under certain conditions may be a phenotype associated with endosomal recycling dysfunction.

Mutations identified in SYS patients are exclusively truncating mutations [23-25]. MAGEL2 is an intronless gene, and thus it escapes nonsense-mediated mRNA decay. Therefore, accumulation of truncated protein is expected. In contrast, a deletion of the entire MAGEL2 gene of paternal origin is associated with milder phenotypes presenting 
as developmental delay but without joint contractures $[27,28]$. MAGEL2 is only expressed from the paternally derived allele and is located in the common deletion region of PWS. PWS is caused by a paternal deletion of approximately $4 \mathrm{Mb}$ in the 15q11-q13 region, which includes MAGEL2 [29]. Therefore, a PWS patient with a common deletion also lacks paternally derived MAGEL2. PWS patients show developmental delay/intellectual disability (DD/ID), neonatal hypotonia, hyperphagia resulting in severe obesity, and hypogonadism [29]. A patient with a deletion of the entire MAGEL2 shows DD/ID and autism but no hyperphagia or hypogonadism $[27,28]$. Therefore, haploinsufficiency of MAGEL2 should partially contribute to the development of DD/ID in PWS patients. Thus, PWS could be categorized as one of the endosomal recycling-related neurodevelopmental disorders. Nevertheless, the phenotype of SYS, including intellectual disability and joint contracture, is more severe than that of PWS. Collectively, MAGEL2 mutations in SYS patients should disturb the function of the MUST complex more intensively than in PWS, in which haploinsufficiency of MAGEL2 may cause mild dysfunction of the MUST complex.

Mutations in USP7 were also identified in patients with developmental delay, altered behavior, and neurologic anomalies including white matter changes [30,31]. Fountain et al. comprehensively described the clinical features of 23 patients [31]. Most patients with a USP7 mutation showed DD/ID of variable range, autism, and hypotonia, whereas dysmorphic features were not conclusive. It is of note that patients with a USP7 mutation showed brain abnormalities associated with white matter volume loss and thinning of the corpus callosum, although gross brain anomalies were not seen. Recently, USP7-related neurodevelopmental disorder was named Hao-Fountain syndrome (HFS). Mutated USP7 was shown to lack activation of the WASH complex and thereby decreased membrane protein levels including M6PR [30]. It is of interest that patients with a mutation in USP7 show phenotypes (intellectual disability, autism, and hypotonia) overlapping with SYS and PWS, suggesting that these disorders share pathological mechanisms in brain [32].

Dysfunction of the MUST complex is likely to cause neurodevelopmental disorders including SYS, PWS, and HFS. The consequence of dysfunction of the MUST complex might be perturbation of the recycling of membrane proteins. Nevertheless, the link between MUST complex and endosomal recycling still remains to be investigated. Moreover, it is not clear whether retromer or retriever is involved because the WASH complex interacts with both retromer and retriever. Nevertheless, as described in the following section, neurodevelopmental disorders caused by dysfunction of retriever tend to show distinct clinical features. Thus, the involvement of retromer in disorders with a dysfunction of the MUST complex deserves to be investigated.

\section{Mouse Models of Retromer Function}

Several mouse models have been established to study retromer function. Homozygous deletion of core subunits of retromer in mice cause lethal phenotypes during embryonic stages [33], suggesting a fundamental significance of retromer. Hemizygous deletion of Vps35 in mice show almost normal phenotypes; however, when these mice are crossed with AD model mice, the resulting double heterozygous mice show earlier development of AD phenotypes including accumulation of $A \beta$ [33]. Therefore, a causal relationship of retromer dysfunction and AD development was suggested in mice studies.

For the relationship between VPS35 and PD, VPS35 D620N knock-in (KI) mice were produced and both heterozygous and homozygous VPS35 D620N mice presented clinical features of PD in an age-dependent manner [34]. VPS35 D620N KI mice showed a decrease in dopamine neurons [34,35]. Unexpectedly, VPS35 D620N KI mice showed robust taupositive neurons, which is related to $\mathrm{AD}$, but lacked the accumulation of $\alpha$-synuclein that is expected for PD. Heterozygous Vps35 null mice or conditional VPS35 KO mice in dopamine neurons did not show the accumulation of tau but presented several features of PD [36,37]. Therefore, loss of function of VPS35 in dopamine neurons induces neurodegeneration and underlies the neuropathology of PD. It is of note that the VPS35 D620N mutation is suggested to have a gain-of-function mechanism [36]. Therefore, precise control of 
membrane proteins by retromer complexes, at least in dopamine neurons, is essential to prevent neurodegeneration, which in turn causes PD.

Two lines of Magel2 knock-out (KO) mouse models have been established [38,39]. One mouse model, which lacked most of the Magel2 gene and its promoter, showed hypothalamic dysfunction, decreased pre-wean weight gain, circadian dysfunction, altered appreciation of novelty, and a mildly increased neonatal lethality [38]. The other mouse model showed phenotypes of disturbed sociability, decreased suckling, decreased oxytocin levels, and approximately $50 \%$ of neonatal lethality [39]. Therefore, the phenotypes of the two mouse models overlapped but were not identical. Therefore, we have introduced a frameshift mutation in Magel2 and created SYS model mice because patients with SYS carry a truncating mutation in paternally derived MAGLE2 [40]. Magel2 is a maternally imprinted gene, and SYS model mice maintained proper imprinting and expression patterns in the brain. Unexpectedly, SYS model mice showed very mild phenotypes compared with those of previously created mouse models and human phenotypes. Ongoing studies of retromer function in the SYS mouse model would reveal the significance of the MUST complex in retromer function.

\section{Human Disorders Associated with Retriever Dysfunction}

Unlike with retromer dysfunction, in patients with a pathogenic mutation in genes associated with retriever function, congenital diseases have been identified. Thus far all mutations are found in patients with similar phenotypes, called Ritscher-Schinzel syndrome (RSS) (Figure 2). RSS is a developmental malformation syndrome characterized by craniofacial abnormalities, congenital heart defects, and cerebellar brain malformation [41]. RSS is also called 3C syndrome representing the craniofacial, cardiac, and cerebellar phenotype although not all features are necessarily present. In 2013, a homozygous mutation in KIAA0196 was first identified in patients with RSS [42]. Elliott et al. reported a large pedigree of ten affected patients with a homozygous splicing mutation in WASHC5. All patients had DD/ID and dysmorphic facies including a prominent forehead, low posterior hairline, wide and downward-slanting palpebral fissures, hypertelorism, and low-set ears. Approximately half of the patients showed cardiac anomalies and brain anomalies including Dandy-Walker malformation and hypoplasia of the cerebellar vermis. Therefore, cardiac or brain anomalies are not present in all patients. KIAA0196 was renamed WASHC5, which encodes strumpellin, a subunit of WASH complex. RSS with a mutation in WASHC5 is called RSS-1.

In 2015, a hemizygous missense mutation in CCDC22 located in Xp11.23 was identified in patients with RSS phenotypes [43]. CCDC22 is a component of the CCC complex, which interacts with retriever. Kolanczyk et al. reported two male siblings who showed DD/ID, facial dysmorphism, cardiac anomaly (ventricular septum defect), and cerebellar malformation (Dandy-Walker malformation). Their facial features included upward slanting palpebral fissures, wide-set eyes, short philtrum, protruding tongue, and a broad neck, making their facial appearance similar to those of RSS1 patients. RSS with a mutation in CCDC22 is named RSS-2.

Lastly, in 2020, Kato et al. reported siblings showing RSS-like phenotypes associated with compound heterozygous mutations in VPS35L, a core subunit of retriever [44]. The siblings presented with DD/ID, craniofacial dysmorphism including prominent forehead, arched eyebrows, downward-slanting palpebral fissures, upturned nose, thin upper lip, and micrognathia. Both siblings had cerebellar malformation (vermis hypoplasia), and one sibling had a cardiac defect (atrioventricular septum defect). Notably, the siblings showed skeletal abnormalities including chondrodysplasia punctata. Their craniofacial dysmorphisms were consistent with those of RSS1 and RSS2, indicating that characteristic craniofacial dysmorphism is specific for RSS. Functional studies revealed that the mutated VPS35L failed to bind to VPS29 and subsequently degraded, clearly leading to a loss of function of the retriever complex. RSS with a mutation in VPS35L is named RSS-3. 
Recently, Jeanne et al. reported nine patients, including one patient from the first family reported by Ritscher et al., and identified missense variants in DPYSL5 [45,46]. A recurrent de novo p.Glu41Lys was found in eight unrelated patients, while a p.Gly47Arg was identified in one patient from the original RSS family. DPYSL5 belongs to the collapsing response mediator protein family and an intracellular mediator of neurotrophic factors regulating neurite structure formation. Therefore, a direct link between DPYSL5 and retriever has not been demonstrated.

Collectively, a deficiency of the retriever-CCC-WASH complex causes neurodevelopmental disorders represented by RSS. Common features of RSS are developmental delay or intellectual disability and characteristic facial features, although other malformations including cardiac defects are associated to varying degrees. Therefore, normal function of retriever endosomal recycling is crucial for brain and craniofacial development as well as for the development of other organs.

\section{Mouse Models for Retriever Function}

Owing to the recent identification of the retriever complex, limited information is available for retriever function in the mouse model. Thus, we introduced a truncating mutation in Vps35l and created model mice [44]. Homozygous Vps35l KO mice died before embryonic day 10.5, suggesting a fundamental role of retriever complex in fetal development. Heterozygous Vps35l showed normal phenotypes corresponding to the human phenotypes. Because of embryonic lethality, it is difficult to test the significance of the retriever complex in development. Thus, conditional $\mathrm{KO}$ mice would be of value to investigate the retriever function in development. Additionally, as in Vps35 KO mice, crosses with AD model mice or other neurodegeneration model mice would be of interest.

\section{Neurodevelopment and Endosomal Recycling}

It is easily understood that proper homeostasis of membrane proteins including receptors and transporters is essential for brain development and function. Thus, defects in endosomal recycling are likely to affect brain development and function. Nevertheless, neurodevelopmental features associated with either retromer dysfunction or retriever dysfunction appear to be distinct, indicating different roles of each system in the brain.

Target membrane proteins of retromer include AMPA and NMDA receptors, which are essential for normal synaptic communications and brain function $[9,10]$. It is of note that compound heterozygous mutations in SNX27, which binds retromer, were identified in patients with epilepsy, developmental delay, and subcortical white matter abnormalities [47]. Patients with homozygous truncating mutations in SNX27 showed more severe phenotypes of infantile myoclonic epilepsy, developmental delay, cardiac defects, and neurodegeneration of progressive neurological deficits [48]. These reports indicated a critical role of retromer in the developing brain, especially in excitatory-inhibitory balance.

Retriever target proteins are numerous among integral proteins required for cell adhesion, including numerous integrins [12]. Cell-cell interaction is crucial in brain development; thus, such difference in target proteins may partly explain the distinct role of retromer and retriever in brain development.

\section{Perspectives of Endosomal Recycle Disorders}

Endosomal recycling dysfunction is growingly recognized as a new entity of neurodevelopmental disorders. Nevertheless, few genes involved in the endosomal recycling system have been identified as causative genes for neurodevelopmental disorders, even though many genes are involved in endosomal recycling. In addition, if we expand our view to the endosomal trafficking system, more and more genes play significant roles. Thus, it is plausible that more genes in endosomal recycling await to be identified as novel causative genes. For the retriever system, the RSS phenotype would be a clue for the identification of such new genes. 
For the retromer system, most genes identified in association with retromer are related to neurodegenerative disorders including AD or PD. SYS or USP7-related disease may be associated with a dysfunction of retromer, although evidence is limited. Given the fundamental roles of retromer in appropriate maintenance of membrane proteins, more genes related to the retromer recycling complex are likely to be associated with neurodevelopmental disorders. The phenotypes of retromer-related neurodevelopmental disorders might not be as specific as those related to retriever.

Endosomal recycling targets numerous membrane proteins, which seemingly complicates developing specific treatment for neurodevelopmental disorders associated with dysfunction of retromer or retriever. Catalogues for dysregulated genes for each disorder would be of value for the development of pathophysiology-based therapeutic interventions. Alternatively, improvement in endosomal recycling through upregulating retromer or retriever function would be a novel therapeutic target for certain neurodevelopmental or neurodegenerative disorders. Indeed, such attempts have been tried for AD. Li et al. introduced AAV-VPS35 into the brain of 3xTg mice, which are model mice developing ADlike neuropathology and behavioral deficits [49]. They found that VPS35 overexpression ameliorated behavioral deficits as well as neuropathology. Therefore, targeting endosomal recycling would be a new paradigm for identifying treatments for neurodevelopmental as well as neurodegenerative disorders.

Funding: This research was partly supported by JSPS KAKENHI Grant Number JP20K21583.

Conflicts of Interest: The author declares no conflict of interest.

\section{References}

1. Uhlén, M.; Fagerberg, L.; Hallström, B.M.; Lindskog, C.; Oksvold, P.; Mardinoglu, A.; Sivertsson, Å.; Kampf, C.; Sjöstedt, E.; Asplund, A.; et al. Proteomics. Tissue-based map of the human proteome. Science 2015, 347, 1260419. [CrossRef]

2. Chen, K.E.; Healy, M.D.; Collins, B.M. Towards a molecular understanding of endosomal trafficking by Retromer and Retriever. Traffic 2019, 20, 465-478. [CrossRef] [PubMed]

3. Anggono, V.; Huganir, R.L. Regulation of AMPA receptor trafficking and synaptic plasticity. Curr. Opin. Neurobiol. 2012, 22, 461-469. [CrossRef] [PubMed]

4. Cullen, P.J.; Steinberg, F. To degrade or not to degrade: Mechanisms and significance of endocytic recycling. Nat. Rev. Mol. Cell Biol. 2018, 19, 679-696. [CrossRef]

5. McNally, K.E.; Faulkner, R.; Steinberg, F.; Gallon, M.; Ghai, R.; Pim, D.; Langton, P.; Pearson, N.; Danson, C.M.; Nägele, H.; et al. Retriever is a multiprotein complex for retromer-independent endosomal cargo recycling. Nat. Cell Biol. 2017, 19, 1214-1225. [CrossRef] [PubMed]

6. Seaman, M.N.; McCaffery, J.M.; Emr, S.D. A membrane coat complex essential for endosome-to-Golgi retrograde transport in yeast. J. Cell Biol. 1998, 142, 665-681. [CrossRef]

7. Lucas, M.; Hierro, A. Retromer. Curr. Biol. 2017, 27, R687-R689. [CrossRef]

8. Chandra, M.; Kendall, A.K.; Jackson, L.P. Toward Understanding the Molecular Role of SNX27/Retromer in Human Health and Disease. Front. Cell Dev. Biol. 2021, 9, 642378. [CrossRef]

9. Steinberg, F.; Gallon, M.; Winfield, M.; Thomas, E.C.; Bell, A.J.; Heesom, K.J.; Tavaré, J.M.; Cullen, P.J. A global analysis of SNX27-retromer assembly and cargo specificity reveals a function in glucose and metal ion transport. Nat. Cell Biol. 2013, 15, 461-471. [CrossRef]

10. Clairfeuille, T.; Mas, C.; Chan, A.S.; Yang, Z.; Tello-Lafoz, M.; Chandra, M.; Widagdo, J.; Kerr, M.C.; Paul, B.; Mérida, I. A molecular code for endosomal recycling of phosphorylated cargos by the SNX27-retromer complex. Nat. Struct. Mol. Biol. 2016, 23, 921-932. [CrossRef]

11. Ghai, R.; Bugarcic, A.; Liu, H.; Norwood, S.J.; Skeldal, S.; Coulson, E.J.; Li, S.S.; Teasdale, R.D.; Collins, B.M. Structural basis for endosomal trafficking of diverse transmembrane cargos by PX-FERM proteins. Proc. Natl. Acad. Sci. USA 2013, 110, E643-E652. [CrossRef]

12. Mallam, A.L.; Marcotte, E.M. Systems-Wide Studies Uncover Commander, a Multiprotein Complex Essential to Human Development. Cell Syst. 2017, 4, 483-494. [CrossRef]

13. Small, S.A.; Kent, K.; Pierce, A.; Leung, C.; Kang, M.S.; Okada, H.; Honig, L.; Vonsattel, J.P.; Kim, T.W. Model-Guided microarray implicates the retromer complex in Alzheimer's disease. Ann. Neurol. 2005, 58, 909-919. [CrossRef]

14. Small, S.A.; Simoes-Spassov, S.; Mayeux, R.; Petsko, G.A. Endosomal Traffic Jams Represent a Pathogenic Hub and Therapeutic Target in Alzheimer's Disease. Trends Neurosci. 2017, 40, 592-602. [CrossRef] [PubMed]

15. Vilariño-Güell, C.; Wider, C.; Ross, O.A.; Dachsel, J.C.; Kachergus, J.M.; Lincoln, S.J.; Soto-Ortolaza, A.I.; Cobb, S.A.; Wilhoite, G.J.; Bacon, J.A.; et al. VPS35 mutations in Parkinson disease. Am. J. Hum. Genet. 2011, 89, 162-167. [CrossRef] [PubMed] 
16. Zimprich, A.; Benet-Pagès, A.; Struhal, W.; Graf, E.; Eck, S.H.; Offman, M.N.; Haubenberger, D.; Spielberger, S.; Schulte, E.C.; Lichtner, P.; et al. A mutation in VPS35, encoding a subunit of the retromer complex, causes late-onset Parkinson disease. Am. J. Hum. Genet. 2011, 89, 168-175. [CrossRef] [PubMed]

17. Zavodszky, E.; Seaman, M.N.; Moreau, K.; Jimenez-Sanchez, M.; Breusegem, S.Y.; Harbour, M.E.; Rubinsztein, D.C. Mutation in VPS35 associated with Parkinson's disease impairs WASH complex association and inhibits autophagy. Nat. Commun. 2014, 5 , 3828. [CrossRef] [PubMed]

18. McGough, I.J.; Steinberg, F.; Jia, D.; Barbuti, P.A.; McMillan, K.J.; Heesom, K.J.; Whone, A.L.; Caldwell, M.A.; Billadeau, D.D.; Rosen, M.K.; et al. Retromer Binding to FAM21 and the WASH Complex Is Perturbed by the Parkinson Disease-Linked VPS35(D620N) Mutation. Curr. Biol. 2014, 24, 1678. [CrossRef] [PubMed]

19. Filippone, A.; Praticò, D. Endosome Dysregulation in Down Syndrome: A Potential Contributor to Alzheimer Disease Pathology. Ann. Neurol. 2021, 90, 4-14. [CrossRef]

20. Hithersay, R.; Startin, C.M.; Hamburg, S.; Mok, K.Y.; Hardy, J.; Fisher, E.M.C.; Tybulewicz, V.L.J.; Nizetic, D.; Strydom, A. Association of Dementia with Mortality Among Adults with Down Syndrome Older Than 35 Years. JAMA Neurol. 2019, 76, 152-160. [CrossRef]

21. Wiseman, F.K.; Pulford, L.J.; Barkus, C.; Liao, F.; Portelius, E.; Webb, R.; Chávez- Gutiérrez, L.; Cleverley, K.; Noy, S.; Sheppard, O.; et al. Trisomy of human chromosome 21 enhances amyloid- $\beta$ deposition independently of an extra copy of APP. Brain 2018, 141, 2457-2474. [CrossRef]

22. Curtis, M.E.; Yu, D.; Praticò, D. Dysregulation of the Retromer Complex System in Down Syndrome. Ann. Neurol. 2020, 88, 137-147. [CrossRef]

23. Schaaf, C.P.; Gonzalez-Garay, M.L.; Xia, F.; Potocki, L.; Gripp, K.W.; Zhang, B.; Peters, B.A.; McElwain, M.A.; Drmanac, R.; Beaudet, A.L.; et al. Truncating mutations of MAGEL2 cause Prader-Willi phenotypes and autism. Nat. Genet. 2013, 45, 1405-1408. [CrossRef] [PubMed]

24. Fountain, M.D.; Aten, E.; Cho, M.T.; Juusola, J.; Walkiewicz, M.A.; Ray, J.W.; Xia, F.; Yang, Y.; Graham, B.H.; Bacino, C.A.; et al. The phenotypic spectrum of Schaaf-Yang syndrome: 18 new affected individuals from 14 families. Genet. Med. 2017, 19, 45-52. [CrossRef] [PubMed]

25. Negishi, Y.; Ieda, D.; Hori, I.; Nozaki, Y.; Yamagata, T.; Komaki, H.; Tohyama, J.; Nagasaki, K.; Tada, H.; Saitoh, S. Schaaf-Yang syndrome shows a Prader-Willi syndrome-like phenotype during infancy. Orphanet J. Rare Dis. 2019, 14, 277. [CrossRef] [PubMed]

26. Hao, Y.H.; Doyle, J.M.; Ramanathan, S.; Gomez, T.S.; Jia, D.; Xu, M.; Chen, Z.J.; Billadeau, D.D.; Rosen, M.K.; Potts, P.R. Regulation of WASH-dependent actin polymerization and protein trafficking by ubiquitination. Cell 2013, 152, 1051-1064. [CrossRef]

27. Kanber, D.; Giltay, J.; Wieczorek, D.; Zogel, C.; Hochstenbach, R.; Caliebe, A.; Kuechler, A.; Horsthemke, B.; Buiting, K. A paternal deletion of MKRN3, MAGEL2 and NDN does not result in Prader-Willi syndrome. Eur. J. Hum. Genet. 2009, 17, 582-590. [CrossRef]

28. Buiting, K.; Di Donato, N.; Beygo, J.; Bens, S.; von der Hagen, M.; Hackmann, K.; Horsthemke, B. Clinical phenotypes of MAGEL2 mutations and deletions. Orphanet J. Rare Dis. 2014, 9, 40. [CrossRef] [PubMed]

29. Cassidy, S.B.; Schwartz, S.; Miller, J.L.; Driscoll, D.J. Prader-Willi syndrome. Genet. Med. 2012, 14, 10-26. [CrossRef]

30. Hao, Y.H.; Fountain, M.D.; Fon Tacer, K.; Xia, F.; Bi, W.; Kang, S.H.; Patel, A.; Rosenfeld, J.A.; Le Caignec, C.; Isidor, B.; et al. USP7 Acts as a Molecular Rheostat to Promote WASH-Dependent Endosomal Protein Recycling and Is Mutated in a Human Neurodevelopmental Disorder. Mol. Cell 2015, 59, 956-969. [CrossRef]

31. Fountain, M.D.; Oleson, D.S.; Rech, M.E.; Segebrecht, L.; Hunter, J.V.; McCarthy, J.M.; Lupo, P.J.; Holtgrewe, M.; Moran, R.; Rosenfeld, J.A.; et al. Pathogenic variants in USP7 cause a neurodevelopmental disorder with speech delays, altered behavior, and neurologic anomalies. Genet. Med. 2019, 21, 1797-1807. [CrossRef]

32. Fountain, M.D.; Schaaf, C.P. Prader-Willi syndrome and Schaaf-Yang syndrome: Neurodevelopmental diseases intersecting at the MAGEL2 gene. Diseases 2016, 4, 2. [CrossRef]

33. Wen, L.; Tang, F.L.; Hong, Y.; Luo, S.W.; Wang, C.L.; He, W.; Shen, C.; Jung, J.U.; Xiong, F.; Lee, D.H.; et al. VPS35 haploinsufficiency increases Alzheimer's disease neuropathology. J. Cell Biol. 2011, 195, 765-779. [CrossRef]

34. Chen, X.; Kordich, J.K.; Williams, E.T.; Levine, N.; Cole-Strauss, A.; Marshall, L.; Labrie, V.; Ma, J.; Lipton, J.W.; Moore, D.J. Parkinson's disease-linked D620N VPS35 knockin mice manifest tau neuropathology and dopaminergic neurodegeneration. Proc. Natl. Acad. Sci. USA 2019, 116, 5765-5774. [CrossRef]

35. Niu, M.; Zhao, F.; Bondelid, K.; Siedlak, S.L.; Torres, S.; Fujioka, H.; Wang, W.; Liu, J.; Zhu, X. VPS35 D620N knockin mice recapitulate cardinal features of Parkinson's disease. Aging Cell 2021, 20, e13347. [CrossRef]

36. Tang, F.L.; Erion, J.R.; Tian, Y.; Liu, W.; Yin, D.M.; Ye, J.; Tang, B.; Mei, L.; Xiong, W.C. VPS35 in Dopamine Neurons Is Required for Endosome-to-Golgi Retrieval of Lamp2a, a Receptor of Chaperone-Mediated Autophagy That Is Critical for $\alpha$-Synuclein Degradation and Prevention of Pathogenesis of Parkinson's Disease. J. Neurosci. 2015, 35, 10613-10628. [CrossRef] [PubMed]

37. Tang, F.L.; Liu, W.; Hu, J.X.; Erion, J.R.; Ye, J.; Mei, L.; Xiong, W.C. VPS35 Deficiency or Mutation Causes Dopaminergic Neuronal Loss by Impairing Mitochondrial Fusion and Function. Cell Rep. 2015, 12, 1631-1643. [CrossRef] [PubMed]

38. Bischof, J.M.; Stewart, C.L.; Wevrick, R. Inactivation of the mouse Magel2 gene results in growth abnormalities similar to Prader-Willi syndrome. Hum. Mol. Genet. 2007, 16, 2713-2719. [CrossRef] [PubMed] 
39. Schaller, F.; Watrin, F.; Sturny, R.; Massacrier, A.; Szepetowski, P.; Muscatelli, F. A single postnatal injection of oxytocin rescues the lethal feeding behaviour in mouse newborns deficient for the imprinted Magel2 gene. Hum. Mol. Genet. 2010, 19, 4895-4905. [CrossRef]

40. Ieda, D.; Negishi, Y.; Miyamoto, T.; Johmura, Y.; Kumamoto, N.; Kato, K.; Miyoshi, I.; Nakanishi, M.; Ugawa, S.; Oishi, H.; et al. Two mouse models carrying truncating mutations in Magel2 show distinct phenotypes. PLoS ONE 2020, 15, e0237814. [CrossRef]

41. Kosaki, K.; Curry, C.J.; Roeder, E.; Jones, K.L. Ritscher-Schinzel (3C) syndrome: Documentation of the phenotype. Am. J. Med. Genet. 1997, 68, 421-427. [CrossRef]

42. Elliott, A.M.; Simard, L.R.; Coghlan, G.; Chudley, A.E.; Chodirker, B.N.; Greenberg, C.R.; Burch, T.; Ly, V.; Hatch, G.M.; Zelinski, T. A novel mutation in KIAA0196: Identification of a gene involved in Ritscher-Schinzel/3C syndrome in a First Nations cohort. J. Med. Genet. 2013, 50, 819-822. [CrossRef]

43. Kolanczyk, M.; Krawitz, P.; Hecht, J.; Hupalowska, A.; Miaczynska, M.; Marschner, K.; Schlack, C.; Emmerich, D.; Kobus, K.; Kornak, U.; et al. Missense variant in CCDC22 causes X-linked recessive intellectual disability with features of RitscherSchinzel/3C syndrome. Eur. J. Hum. Genet. 2015, 23, 720. [CrossRef]

44. Kato, K.; Oka, Y.; Muramatsu, H.; Vasilev, F.F.; Otomo, T.; Oishi, H.; Kawano, Y.; Kidokoro, H.; Nakazawa, Y.; Ogi, T.; et al. Biallelic VPS35L pathogenic variants cause 3C/Ritscher-Schinzel-like syndrome through dysfunction of retriever complex. J. Med. Genet. 2020, 57, 245-253. [CrossRef]

45. Jeanne, M.; Demory, H.; Moutal, A.; Vuillaume, M.L.; Blesson, S.; Thépault, R.A.; Marouillat, S.; Halewa, J.; Maas, S.M.; Motazacker, M.M.; et al. Missense variants in DPYSL5 cause a neurodevelopmental disorder with corpus callosum agenesis and cerebellar abnormalities. Am. J. Hum. Genet. 2021, 108, 951-961. [CrossRef]

46. Ritscher, D.; Schinzel, A.; Boltshauser, E.; Briner, J.; Arbenz, U.; Sigg, P. Dandy- Walker(like) malformation, atrio-ventricular septal defect and a similar pattern of minor anomalies in 2 sisters: A new syndrome? Am. J. Med. Genet. 1987, 26, 481-491. [CrossRef]

47. Parente, D.J.; Morris, S.M.; McKinstry, R.C.; Brandt, T.; Gabau, E.; Ruiz, A.; Shinawi, M. Sorting nexin 27 (SNX27) variants associated with seizures, developmental delay, behavioral disturbance, and subcortical brain abnormalities. Clin. Genet. 2020, 97, 437-446. [CrossRef]

48. Damseh, N.; Danson, C.M.; Al-Ashhab, M.; Abu-Libdeh, B.; Gallon, M.; Sharma, K.; Yaacov, B.; Coulthard, E.; Caldwell, M.A.; Edvardson, S.; et al. A defect in the retromer accessory protein, SNX27, manifests by infantile myoclonic epilepsy and neurodegeneration. Neurogenetics 2015, 16, 215-221. [CrossRef]

49. Li, J.G.; Chiu, J.; Praticò, D. Full recovery of the Alzheimer's disease phenotype by gain of function of vacuolar protein sorting 35. Mol. Psychiatry 2020, 25, 2630-2640. [CrossRef] 